\title{
¿Bioética narrativa o narrativa bioética?
}

\section{Narrative bioethics or fictional narrative of bioethical issues?}

\section{Bioética narrativa ou narrativa Bioética?}

Fecha de recepción: 27 de enero de 2016

Fecha de evaluación: 18 de marzo de 2016

Fecha de aceptación: 13 de abril de 2016

Publicación en línea: 16 de abril de 2016

Miguel Kottow*

Doi: http://dx.doi.org/10.18359/rlbi.1763

Cómo citar:

Kottow, M. (2016). ¿Bioética narrativa o narrativa bioética? Revista Latinoamericana de Bioética, 16(2), 58-69. DOI: http://dx.doi.org/10.18359/rlbi.1763

\footnotetext{
* Artículo de Reflexión.

** Profesor Titular de la Universidad Central de Chile. Correo electrónico miguel.kottow@ucentral,cl, mkottow@gmail.com. Fono móvil 993069977. FAX 223310032. ORCID: http://orcid. org/0000-0002-6403-133 Santiago de Chile. Chile.
} 


\section{Resumen}

Desde los inicios de la bioética, se presenta y agudiza el conflicto entre biomedicina científica basada en evidencia y la bioética preocupada de valores personales y participativos expresados en el consentimiento informado. La medicina terapéutica y la investigación biomédica propician la participación autónoma de pacientes y probandos, la cual se expresa en la narrativa que destaca los valores existenciales de cada persona y el contexto social donde vive. Las humanidades médicas son convocadas para sensibilizar a los profesionales de la salud en sus tareas. Su influencia por elevar los elementos narrativos en la comunicación biomédica ha sido insuficiente y de influencia decreciente, su aporte ancilar a la bioética narrativa no ha logrado evitar un academicismo extremo y una irrelevancia social de la disciplina bioética que está en riesgo de ser marginada, desoída y precipitada en una crisis de validación.

Sugiere este artículo atender fuentes no académicas que se muestran plenamente competentes y atractivas para encarar los problemas que la bioética no ha sabido resolver, destacando la publicación de novelas ficcionales que abordan temas bioéticos de forma amena y sistemática, que impactan más allá de las deliberaciones anémicas de la bioética: The Children Act (I. McKewan, 2014) y Reparar a los vivo (M. de Kerangal, M., 2015) son presentadas como propuesta de inaugurar la narrativa bioética, que es el relato ficcional de temas bioéticos. Palabras clave: humanidades médicas, medicina narrativa, bioética narrativa, narrativa literaria de temas bioético.

\section{Abstract}

The beginnings of bioethics faced the conflict between scientifically orientated biomedicine based on evidence, and the bioethical concern with personal values in informed consent, to sustain an interpersonal relationship in clinical encounters. Therapeutic medicine and biomedical research contribute to the free participation of patients and probands, which is expressed in the narrative that highlights the existential values of each person and the social context where they live. The medical humanities have been called upon to assist the healthcare professions to engage in narrative communication, by developing medical and bioethical narratives. Its influence to raise the narrative elements in biomedical communication has been inadequate and declining influence; their ancillary tasks to develop a bioethical narrative have been unable to revive bioethics steeped in academicism and social irrelevance, to the point where the discipline is seen to be in a crisis of validation.

This article explores non-academic literary sources that treat with competence and amenity the issues that bioethics has been unable to solve. Two recently published novels are discussed: I. McKewan's The children act, and M. de Kerangal's Spanish version of Réparer les vivants, both books presenting a little-explored way of treating bioethical issues in a fictional form.

Keywords: Medical humanities, narrative medicine, narrative Bioethics, fictional narrative of bioethical issues.

\section{Resumo}

A partir dos inícios da bioética, apresenta-se e aguça o conflito entre biomedicina científica baseada em evidências e a bioética preocupada de valores pessoais e participativos expressos no consentimento informado. A medicina terapêutica e a investigação biomédica favorecem a participação autônoma de pacientes e provandos, o que se expressa na narrativa que destaca os valores existenciais de cada pessoa e o contexto social em que vivem. As humanidades médicas são convocadas para sensibilizar aos profissionais da saúde em seu trabalho. Sua influência para levantar os elementos narrativos na comunicação biomédica tem sido inadequada e de influência decrescente, a sua contribuição subordinada à bioética narrativa não tem conseguido impedir um academicismo extremo e uma irrelevância social da disciplina bioética que está em risco de ser marginalizada, ignorada e precipitada numa crise de validação.

Este artigo sugere atender fontes não acadêmicas que se mostram plenamente competentes e atraentes para enfrentar os problemas que a bioética não tem sabido resolver, com destaque para a publicação de novelas de ficção que tratam de questões bioéticas em um ambiente agradável e sistemática, que impactam além das deliberações anêmicas da bioética: The Children Act (I. McKewan, 2014) e Reparar a los vivos (M. de Kerangal, M., 2015) são apresentados como uma proposta de inaugurar a narrativa bioética, que é a narração fictícia de questões bioéticas. Palavras-chave: humanidades médicas, medicina narrativa, bioética narrativa, narrativa literária de questões bioéticas. 


\section{Introducción}

$\mathrm{Al}$ acelerarse la vertiginosa carrera de la medicina hacia el rigor tecnocientífico de la biomedicina que amalgama investigación biológica con práctica médica basada en evidencia, se inició con André Hellegers y el Kennedy Institute of Ethics la preocupación por destacar los derechos de participación y decisión del paciente. Poco antes del nacimiento de la bioética, Joseph Fletcher citaba al médico George Jacoby, quien entendía la ética médica como dedicada a la "cuestión de la actitud general del médico hacia el paciente" (Fletcher, 1979, p. 6). El primer capítulo de su libro concluía con la revelación de que "sin la libertad de elegir y el derecho de conocer la verdad, los pacientes no son sino muñecos" (p. 33).

Dejando de lado la bioética global iniciada por V.R. Potter, la vertiente ético-médica de la naciente disciplina colocó en el centro de sus preocupaciones la relación médico-paciente y el despliegue del consentimiento informado como el instrumento más acabado para la cabal participación de pacientes y probandos en las decisiones sobre exploración e intervención en su cuerpo con fines terapéuticos y científicos (Faden y Beauchamp, 1986). El consentimiento informado se basa en desplegar los relatos que relevan los pliegues de la psicología, la sociología, las convicciones y el imaginario, que dan vida a los valores que impregnan la existencia individual y sus relaciones; también está encargado de situarse en forma medular en situaciones médicas, en el espesor de la existencia del paciente, del terapeuta y de todos los involucrados. Sin embargo, el consentimiento informado se ha degradado, pues ha pasado de ser un proceso interpersonal a ser un procedimiento institucional, inhibiendo la narrativa -anamnesis, diálogo paciente-médico-, medular a la práctica médica de todos los tiempos.

Al tiempo que la medicina se fue enfrascando cada vez más en la evidencia científica y en el despliegue de sofisticados métodos de diagnóstico y terapia, la narrativa golpeaba a las puertas de la educación médica, solicitando ingreso mediante apertura hacia las humanidades médicas en general y la literatura en particular. El acompañamiento de la bioética, por su parte, ha sido más irresoluto al verse la disciplina convocada a comentar los despliegues de la tecnociencia y sus consecuencias sociales. En vez de recuperar la veta holística de Potter (1971) que fue a engrosar la deliberación ecoética, comenzó la bioética a ser definida:

[...] en relación a las formas de I + $\mathrm{D}$ en las que el carácter tecnocientífico es muy fuerte y mediante las cuales se ha puesto de manifiesto el carácter experimentalista, pero también manipulador e intervencionista, activo y técnicamente armado de la ciencia contemporánea. (Hottois, 1991, p. 172) 


\section{Narrativa y humanidades}

Desde la filosofía, Paul Ricoeur (2001) llama a centrar la narrativa en la práctica médica:

El paciente (este paciente) <porta en el lenguaje> su sufrimiento al expresarlo como queja que conlleva un componente descriptivo (tal síntoma...) y un componente narrativo (un individuo entramado en tales y tales historias); a su vez, la queja se precisa como una demanda de [...] (sanación y, quién sabe, de salud y, por qué no, al plan de fondo de inmortalidad) y demanda a [...] dirigida como un llamado a tal médico. (pp. 229 y 230)

Tediosos debates sobre sentido y significación de las humanidades minan el respeto social y fuerza académica necesarios. La academia explícitamente dedicada a las humanidades está siendo reducida, asfixiada y desintegrada (Nussbaum, 1997; Small, 2013). En rigor, las humanidades deben entenderse menos como aportando conocimientos que "expandiendo la imaginación moral"; su misión universitaria es distinguir lo pensado "en generaciones previas y en otras clases sociales" (Rorty, 1999, pp. 127-130). La tarea de las humanidades es fomentar el pensamiento crítico que permite analizar con cierta agudeza la realidad, a fin de pensar y proponer valores que enriquezcan el bien común para sustentar mejor los proyectos existenciales de cada uno (Brooks, 2014).
El énfasis del humanismo en narrativas de vida, en el enriquecimiento de vivenciar diferencias, y en la evaluación de herramientas en términos de lo humano más que en la complejidad mecánica, son todos valores vitales -y aún más, diría-, son ponderaciones críticas del estado de la sociedad moderna. (Sennet, 2011, p. 30)

Mientras ascendía la influencia de la bioética enfrascada en disquisiciones sobre genética, reproducción asistida, clonación, investigación biomédica, se debilitan las humanidades infructuosamente empeñadas en ganar espacio curricular en los estudios superiores y hacerse presente en la formación del médico. Es efectivo que la bioética siguió manteniendo interés en el consentimiento informado, insistiendo en perfeccionar y ampliar el ámbito de comunicación, mencionando la dependencia cultural, situacional y personal en su práctica, pero sin intentos por sistematizar la diversidad. Respetando la distinción entre lo que el paciente siente (síntomas) y lo que la exploración de su cuerpo muestra (signos) y aplicando las reflexiones del filósofo del lenguaje Mikhail Bakhtin (1895-1975), se reconoce que el relato inicial del paciente es polifónico, conteniendo elementos sociales, opiniones y convicciones que han emergido en relación con este episodio mórbido en particular. El receptor médico reduce el relato matizado del paciente a un "todo sistemáticamente monologizado" (Bakhtin, 1997, citado en Puustinen, 1999, p. 279), interpretación que en la 
biomedicina tecnificada puede llevar (de hecho lleva) a una tan extrema reducción que convierte al paciente -también en palabras de Bakhtin- en un "objeto sin voz". El lenguaje médico de retorno es asimismo polifónico, pero expresado de forma escueta como un diagnóstico y un tratamiento dirigido al cuerpo enfermo, no al paciente existencialmente comprometido.

El desarrollo de las perspectivas "no médicas", de las asignaturas "blandas", del currículum oculto en los programas de pre y posgrado en las escuelas de medicina terminó siendo encomendado a la bioética, que se encontró sobrepasada, excesivamente exigida, imposibilitada de hacer justicia a las humanidades y la disciplina bioética (Fox, 1999). La solución parecía residir en el desarrollo de un programa de humanidades médicas, manteniendo la bioética como un ciclo $o$ asignatura.

\section{Humanidades médicas y bioéticas}

"Como disciplinas académicas, historia, literatura o filosofía han de ser vistas simplemente como componentes de una educación bien redondeada. ¿Desean los médicos ser bien educados o simplemente entrenados en forma estrecha?" (Downie, 2003, p. 37) (la traducción es mía).

La indeterminación del campo de las humanidades médicas dificulta enmarcar su espacio académico y curricular, lo que hace imposible bregar frente a las llamadas "disciplinas duras" de la educación médica.

Una condición poco delimitada de preocupaciones, personas, disciplinas, aproximaciones, prácticas y métodos comprometidos en un diálogo relativamente abierto, explorando donde los enfoques de la humanidades etc. (sic) pueden ser esclarecedoras de, o incluso obstructivas a, la salud y los cuidados de la salud. (Pattison, 2003, p. 33)

Claramente, el discurso sobre humanidades en general y su importancia en la educación superior en particular no han logrado convencer de su especificidad y efectividad en la formación en las profesiones de la salud. La extensa medicalización de nuestras sociedades, así como la centralidad de la economía de la salud, viene dejando cada vez menos espacio a disciplinas que no son positivistas, métricas, científicas. La perspectiva humanista junto con la bioética han debido sumergirse en las prácticas sociales institucionalizadas de la medicina, de la investigación biomédica, relevando su importancia para incrementar la calidad y eficiencia de estas prácticas; al mismo tiempo uniéndose al coro de reclamos por la deshumanización de la medicina que, más en rigor, debiera ser vista como una despersonalización. El acto médico no es inhumano, es humanamente desprolijo y desatento a la persona enferma. 


\section{Medicina narrativa}

Los relatos se engarzan en la práctica ética de la medicina de dos modos: por su contenido y por "su forma diegética, esto es, por su comprensión del modo cómo son narrados y por qué ello es importante" (Jones, 1999, p. 253). Los relatos ejemplares complementan la enseñanza de principios ético-médicos, pretenden ser guías para la buena vida, el cultivo de valores en aras de una práctica médica virtuosa y, en tercer término, como narrativas testimoniales y autobiográficas. El análisis literario de narrativas médicas ha sido de más interés disciplinar que de utilidad para la medicina, lo cual contribuyó a mermar su influencia en la formación médica.

La sustitución en el curso de la formación médica de habilidades estimadas como "científicas" -aquellas que son eminentemente mensurables pero inevitablemente reduccionistas- en desmedro de lo fundamentalmente lingüístico, empático e interpretativo, ha de verse como todo menos un rasgo exitoso del currículo moderno. (Greenhalgh y Hurwitz, 1999, p. 50)

El acto médico se basa en una comunicación entre paciente y terapeuta, y requiere también la interacción comunicativa del sistema médico con sus componentes, el lenguaje científico y el asistencial. Lo que se comunica es un relato, una narración que le otorga significación a los datos, conecta vivencias e información con decisión y acción. La narrativa permea la enfermedad, "la his- toria que cada uno de nosotros se cuenta a sí mismo en estado de minoración", la historia que el enfermo "compone para interesar a otros y reclamar su ayuda", la historia que el médico presenta en forma de diagnóstico (Lolas, 1991 p. 87).

Las humanidades, la literatura, la lecturas de textos relacionados con enfermedad y medicina contribuyen a la adquisición de competencias narrativas, son fuentes para perfeccionar la "medicina narrativa, entendida como la medicina practicada con las habilidades de reconocer, absorber, interpretar y sentirse movido por las historias de enfermedad". (Charon, 2006, p. 4)

\section{Bioética narrativa}

En una primera aproximación, la bioética narrativa propicia, de forma similar a la medicina narrativa, un enriquecimiento de la comunicación a objeto de precisar la deliberación médica en plantear un diagnóstico y aplicar la terapia más eficaz; para la bioética, la deliberación es de orden ético a objeto de esclarecer valores y asistir en la toma de decisiones. La deliberación bioética es tanto más necesaria cuando se requiere "desarrollar conceptos éticos alternativos ante las manchas ciegas de una ética basada únicamente en principios" (Dubiel, 2011, p. 10). La bioética narrativa se suma a las iniciativas incomodadas por la rigidez de principialismos explícitos, teniendo una comunidad de intenciones con perspectivas bioéticas más dúctiles 
como el casuismo, el feminismo, la ética de cuidados: "Puesto que la bioética principialista emergió para encarar relaciones clínicas oposicionales, no se puede esperar de ella que incremente las relaciones de cuidados" (Charon, 2006, p. 208). Charon propone que los problemas éticos intrínsecos a la práctica médica no requieren una función bioética adicional, puesto que en la "bioética narrativa encontramos el respeto de lo que he llamado los tres movimientos de la medicina narrativa -atención, representación y afiliación-" (2006, p. 211). No obstante, "[L]o que falta en bioética así como en la práctica clínica es precisamente la perspectiva que se vuelve posible a través de una sofisticada práctica narrativa, especialmente [...] en las área de temporalidad, singularidad, intersubjetividad, causalidad/contingencia y eticidad" (Charon, 2006, p. 212).

En tanto la ética era aplicada a prácticas sociales, la bioética se entraba en conflictos y generaba antagonismos principalmente en el ámbito de la investigación con seres humanos, mas también en la práctica clínica, donde no es abiertamente desautorizada sino simplemente ignorada. "Afuerinos se introdujeron en la medicina para corregir lo que percibían como errores [maleficentes] -wrongs-, indispuestos a aceptar los potenciales beneficios sociales y la negociación del interés individual. En suma, encontraron daño donde los investigadores percibieron la oportunidad de progreso" (Rothman, 1991, p. 94).
La crítica a la medicina es transversal, pero las propuestas remediales difieren y son conflictuales. La literatura descalifica a la bioética o pone en duda la solvencia de "afuerinos" para opinar sobre prácticas médicas y protocolos de investigación cuyas insuficiencias y deficiencias han de ser resueltas en su interior, en la tradicional postura que la profesión médica ha de ser su propio fiscal ético.

\section{Crisis estancada}

"Una revisión de las más recientes publicaciones en bioética muestra claramente que la disciplina está sumida en una profunda crisis" (Maldonado, 2012, p. 114) (la traducción es mía).

El anterior es un gravísimo diagnóstico que se vincula directamente a una de las principales metas de la bioética en sus etapas iniciales de "proto-bioética" (Pellegrino, 1999, p. 74):

Lo que hoy se conoce por "bioética" comenzó una década y media después del término de la $2^{\mathrm{a}}$ Guerra Mundial, como un apenas definido movimiento por "humanizar" la educación y la práctica médica. El objetivo era tamponar y equilibrar el poderoso movimiento hacia la especialización y hacia la ciencia que comenzaba, ya entonces, a dominar la formación de los médicos. (p. 75)

Se repite insistentemente que la medicina se ha deshumanizado de forma acelerada desde mediados del siglo pasado, y 
ahora ha de reconocerse que el encargo a la bioética por revertir este proceso no ha dado frutos y se encuentra, a su vez, en crisis. En otras palabras, la medicina sufre de una crisis de humanización que la bioética ha sido incapaz de afrontar. El concepto crisis se refiere a una situación de severa disfuncionalidad, pero que tiende a movilizarse, ora hacia un agravamiento demoledor, ora hacia la mejoría. En su primigenia aplicación en la medicina hipocrática, la crisis de una enfermedad era el momento - el día crítico- en que el paciente se agrava y muere, o inicia un curso de recuperación y sanación. Las crisis son situaciones pasajeras, pero la crisis de la medicina, relevada por Illich, Ongaro Basaglia y tantos otros, es un estado de crónica profundización.

A diferencia de ello, la bioética mantiene esfuerzos por hacerse cargo y sustentar los valores involucrados en las prácticas sociales que le incumben: medicina, investigación biomédica, salud pública, ecología. Uno de los principales impulsos de renovación ha sido el énfasis en la narrativa y el cultivo de las humanidades para sensibilizar a agentes y pacientes en la presentación, interpretación e interlocución de narraciones. No obstante, estos esfuerzos no han rendido los frutos esperados, y han perdido así importancia y presencia en un mundo pragmático concentrado en eficiencia y cumplimiento de metas. Arrecian las críticas contra la bioética -Baron-, su incompetencia filosófica-Savulescu-y su irrelevancia social -Hedgecoe-.

\section{Narrativas bioéticas}

Los conflictos bioéticos han concitado el interés de la literatura narrativa, construyendo mundos de seres clonados (Houllebeck, Ishiguru), titilando la imaginación futurista en la descripción de un posible mundo donde la tecnociencia tuerce los procesos naturales y crea cíborgs, posthumanos, quimeras. Este tipo de narrativa continúa los clásicos mitos y relatos de Pigmalión, Frankenstein, Dorian Grey, entre otros, que en la deliberación bioética contemporánea tienen uso metafórico ilustrativo, pero que no guían en la resolución de conflictos.

Más interesante es la muy reciente aparición de novelas cuya trama es el conflicto bioético, que es narrativamente explotado y llevado a alguna resolución, lo cual despliega un relato cautivante y provisto de dénouement, contrastando con mucha deliberación bioética que se ha vuelto tediosa-Jonsen-e infructuosa.

\section{The children act, de Ian McKewan}

Fiona Maye es jueza de la High Court ${ }^{1}$, especializada en juicios de familia. Su matrimonio está en una crisis, desencadenada la víspera de una sentencia que debe dictar en un caso de dos hermanas de convicción Hasedi, cuyos padres separados difieren respecto a si sus hijas deben recibir una educación religiosa o, según disputa la madre, atender una escuela moderna, coeducacional,

\footnotetext{
1 Corte superior a la par con la Cortes de
} Apelaciones y la Corte de la Corona. 
más apropiada para su socialización en un mundo secularizado. Pasa por su mente un reciente caso de siameses que morirían si no eran separados, aunque invariablemente no sobreviviría el más débil en el acto quirúrgico que salvaría al otro. Una situación que la jueza resolvió recurriendo a la "doctrina de necesidad", una variante judicial de la doctrina de doble efecto: salvar a un niño a sabiendas que el otro morirá.

Mientras prepara su sentencia que dará razón a la madre en conceder a sus hijas una educación libre de la postura ortodoxa que forma a la mujer para alcanzar su plenitud en el hogar, la jueza recibe una llamada en nombre de un hospital que solicita apoyo judicial para transfundir a un muchacho de 17 años quien, junto con sus padres, rechaza la transfusión por ser testigo de Jehová.

El joven Adam está en tratamiento por leucemia complicada con anemia aplástica y por la urgencia de una transfusión de sangre sin la cual morirá en breve. La jueza solicita información detallada sobre el caso médico, las indicaciones, riesgos y eventual futilidad de la transfusión. El paciente y sus padres son inflexibles en negar el procedimiento; el hijo está a tres meses de la mayoría de edad para decidir, aunque no cabe duda de su competencia mental, siendo descrito como una persona muy inteligente, escritor de notables poesías.

Después de oír los argumentos de los médicos, los padres y los representantes de los testigos de Jehová, la jueza decide visitar a Adam, conocer la profundidad de sus creencias y la autenticidad de su disposición a morir. Dada la urgencia del caso, debe haber un dictamen judicial ese mismo día:

En suma, considero que A, sus padres y los consejeros de la iglesia (Elders Mayores) han tomado una decisión que es hostil al bienestar de $\mathrm{A}$, que es la consideración principal de la corte. Él debe ser protegido de tal decisión. Él debe ser protegido de su religión y de sí mismo.

Este asunto no ha sido fácil de resolver. He otorgado debida ponderación a la edad de A, el debido respeto a su fe y a la dignidad del individuo inherente al derecho de rechazar tratamiento. A mi juicio, su vida es más preciosa que su dignidad. (McKewan, 2014, p. 127)

Quedan judicialmente sobreseídos los rechazos al aceptar la medida cautelar del hospital y proceder a la transfusión de sangre.

\section{Reparar a los vivos, de Maylis de Kerangal}

A la edad de 19 años, Simón Limbres queda en estado de muerte cerebral tras un accidente en automóvil. El médico de turno informa a la madre que su hijo está en "un coma profundo", luego "Notifica que ha iniciado el trámite legal destinado a confirmar la muerte del joven [...] angiografía por escáner, radiografía [...] la realización de dos EEG de treinta 
minutos, realizados con un intervalo de cuatro horas" (Kerangal, 2015, p. 71).

El enfermero especializado en desarrollar el protocolo de trasplante de órganos "capta el mensaje y declara: entonces podremos proceder a una evaluación completa de los órganos" (p. 71).

Reunión de médico, enfermero, los padres de Simón, una escena delicadamente descrita que se inicia con la frase "Nos hallamos en un contexto en el que cabría la posibilidad de que Simón donase sus órganos" (p. 103). Los padres se niegan, no habrá donación aunque la ley francesa opera con el consentimiento presunto, porque el enfermero solicitante "ha asumido el principio de respetar por entero la opinión de los familiares" (p. 111). Los padres se retiran, cavilan, deliberan entre ellos y cada uno consigo mismo, vuelven al hospital y resuelven: "Donará".

La segunda mitad de la novela es un relato cinematográfico del proceso de organización del rescate de órganos; su expedito traslado a hospitales cercanos y distantes, una minuciosa descripción de las técnicas de obtención, conservación y trasplante multiorgánico: el corazón queda en París, los pulmones van a Lyon, Estrasburgo recibe el hígado, Rouen, los riñones. Los ojos no, el padre se niega a que sean extraídos. El relato lleva al lector a los pabellones quirúrgicos, el transporte aéreo y terrestre, la preparación de los equipos médicos, todo relatado con precisión testimonial y, novela al fin, entreteje el relato narrando episodios de la existencia de varios protagonistas. Ya están tomadas las decisiones de la donación, todo el despliegue está normado y protocolizado en todos sus detalles, ilustrando cómo la eficiencia de la medicina del trasplante depende de una organización acabada, tanto o más que los avatares legales, personales y familiares de la disposición a donar.

\section{Conclusión}

Se presentaron dos ejemplos de literatura narrativa que se tejen magistralmente en torno a temas de indudable relevancia bioética: decisiones terapéuticas contra la voluntad del paciente y sus padres, con la audaz decisión de sobreponer la vida de un ser humano por sobre su dignidad; en el segundo caso, "[E]nterrar a los muertos y reparar a los vivos", la reflexión medular que inspira la ponderación valórica de donar órganos o mantener inmaculado el cadáver de un ser querido aun a costa de negar asistencia a receptores necesitados. La ley que consiente, la medicina del trasplante que respeta la voluntad de donar que ha de imperar sobre lo legal.

La lectura de estas obras - probablemente existan otras- permite inaugurar una vertiente de esclarecimiento donde la bioética pasa a ser adjetivo de la literatura: narrativa bioética. Es una llamada de alerta a reflexionar cómo los temas que ocupan a la bioética y solazan a sus académicos son cabalmente abordables sin recurrir a la especialización disciplinaria. El imaginario narrativo sabe 
retratar una medicina "humanizada" con más prestancia que la desgastada "estrechez de visión y tendencia a conservadurismo de la bioética" (Brody, 2009, p. 40). Ninguno de estos textos siquiera menciona la bioética ni sus instrumentos deliberativos y resolutivos -comités, comisiones, expertos-.

El enfoque narrativo y la literatura como parte de las humanidades médicas han sido como "las más pertinentes como esenciales a la bioética", tanto en forma de narrativa ética que se opone al principialismo, como "narrativas que contribuyen a la bioética". La narrativa bioética aquí relevada no es meramente contributiva, sino que aborda con imaginación y resolución materias que siguen entrampadas en la bioética, insinuando que la narración literaria puede reemplazar a la disciplina bioética y marginarla como un ejercicio académico infértil.

Tal como la medicina científica pierde confiabilidad y es reemplazada por terapias naturales y medicinas alternativas, también la bioética ha perdido influencia y está en riesgo de un estancamiento reduccionista y utilitarista, esterilizada por un quehacer sumido en un estado de crisis que ha sido paliado pero no superado, recurriendo a la narrativa como función ancilar. La imaginación narrativa muestra que los temas de la bioética deben acercarse al sentido común, a la relevancia social y mostrar, si puede, la inventiva y resolución de un relato ficcional, aun cuando está basado en la realidad.
Es un esfuerzo por frenar la "deshumanización" de la medicina, su "despersonalización "y recuperar para los derechos humanos su espíritu humanitario en vez de someterlos a los avatares de la política contingente. La bioética, como también la filosofía, hace un llamado por encarnar los valores en curso en una narrativa que sitúe las vivencias existenciales por sobre la mercantilización y el utilitarismo de todos los procesos sociales de bienes y servicios. Lo que medicina y bioética narrativa no han logrado, tal vez pueda fructificar por medios de la narrativa bioética.

\section{Referencias}

Brody, H. (2009). The Future of Bioethics. Oxford: Oxford University Press.

Brooks, P. (Ed.) (2014). The Humanities and Public Life. New York: Fordham University Press.

Charon, R. (2006). Narrative Medicine. Oxford: Oxford University Press.

Downie, R. S. (2003). Medical humanities: a vision and some cautionary notes. Medical humanities, 29, 37-38.

Dubiel, H. (2011). What is "Narrative Bioethics". Frontiers in Integrative Neuroscience, 5, DOI: 10.3389/fnint.2011.00010.

Faden, R. R. y Beauchamp, T. L. (1986) A history and theory of informed consent. Oxford: Oxford University Press.

Fletcher, J. (1979). Morals and medicine. Princeton: Princeton University Press.

Fox, R. C. (1999). Is medical education asking too much of bioethics? Daedalus, 128(4), 1-25. 
Greenhalgh, T. y Hurwitz, B. (1999). Why study narrative? British Medical Journal, 318(2), 48-50.

Hottois, G. (1991). El paradigma bioético. Barcelona: Anthropos.

Jones, A. H. (1999). Narrative in medical ethics. British Medical Journal, 318(2), 253-256.

Kerangal, M. de (2015). Reparar a los vivos. Barcelona: Anagrama.

Lolas, F. S. (6 de diciembre de 1991). La medicina como narrativa. Discurso Incorporación Academia Chilena de la Lengua. (Apartado). Revista Médica de Chile, 120, 810-815.

Maldonado, C. E. (2012). Crisis of bioethics and bioethics in the midst of crises. Revista Latinoamericana de Bioética, 12(1), 112-123.

McKewan, I. (2014). The children act. New York: Doubleday

Nussbaum, M. C. (1997). Cultivating Humanity. Cambridge: Harvard University Press.
Pattison, S. (2003). Medical humanities: a vision and some cautionary notes. Medical humanities, 29, 33-35.

Pellegrino, E.D. (1999). The Origins and Evolution of Bioethics. Kennedy Institute of Ethics Journal, 9(1), 73-88.

Puustinen, R. (1999). Bakhtin's philosophy and medical practice-Toward a semiotic theory of doctor - patient interaction. Medicine, Health Care and Philosophy, 2(3), 275-281.

Ricoeur, P. (2001). Le Juste 2. Paris: Éditions Esprit.

Rorty, R. 1999). Philosophy and Social Hope. London: Penguin Books.

Rothman, D. J. (1991). Strangers at the bedside. New York: Basic Books.

Sennet, R. (2011). Humanism. The Hedgehog Review / Summer, 21-30.

Small, H. (2013). The Value of the Humanities. Oxford: Oxford University Press. 